\title{
The finite element method for flexure of slabs when stress distributions are assumed
}

by

\author{
R. T. Severn, B.Sc., Ph.D.
}

and

\author{
P. R. Taylor, B.Sc.(Eng.), Ph.D.
}

Mr I. M. Smith (Assistant Lecturer, Department of Civil Engineering, University of Glasgow) wrote that the Authors made some categorical statements with which he did not agree. In the synopsis they stated that 'It is well known that when displacement distributions are assumed, the solutions obtained converge to the correct values from above as the element size is reduced.' If by 'solutions' the Authors meant displacements as they subsequently implied by references to Table 1, then he thought that that statement was incorrect. For an assumed displacement distribution which did not ensure complete interelement compatibility, such as the distribution assumed by Zienkiewicz and Cheung, ${ }^{2}$ for example, one could not be sure from which 'side' the solution would converge. For certain problems the convergence was from below while for others it was from above. Even if complete interelement compatibility was ensured, the displacements of all points on a plate structure might not be underestimated. ${ }^{7}$ Therefore, he felt it was rather unlikely that the "mixed' approach which the Authors had used would lead to consistent underestimates of the deflexions of plates.

21. The Authors went on to state ( $(15)$ that 'A matter of great importance in the finite element method is the fact that at a junction between two elements discontinuities in bending moment do exist.' Again Mr Smith thought that was not necessarily so, and it was possible to develop a displacement distribution which ensured the continuity of $\partial^{2} w / \partial x^{2}, \partial^{2} w / \partial y^{2}$, and $\partial^{2} w / \partial x \partial y$ between adjacent elements, ${ }^{8}$ and hence gave continuity of bending moment.

22. The above comments did not detract from the main content of the Paper, and the results given by the Authors were impressively good. Mr Smith would be interested in the solutions by the Authors' method to other problems, for example the simply supported square plate carrying a uniformly distributed load.

Mr R. G. Tickell (G. Maunsell and Partners) wrote that the Authors were to be congratulated on their lucid Paper which extended the versatility of the finite element method; it was a useful tool for the elastic analysis of the complex slabs, which were occurring with greater frequency in current practice.

24. The first point which should be made was that the rule for matrix multiplication appeared to be violated in equation (8) and several similar equations. Equation (8) should surely read

$$
U=\frac{1}{2}\{\beta\}^{\mathrm{T}}[\mathbf{H}]\{\beta\} \text {. }
$$

25. The impression was given that in developing the stiffness matrix for the unit element one could either assume a displacement form for the element or a stress

* Proc. Instn civ. Engrs, 1966, 34 (June) 153-170.

7 The references appear on p. 705. 


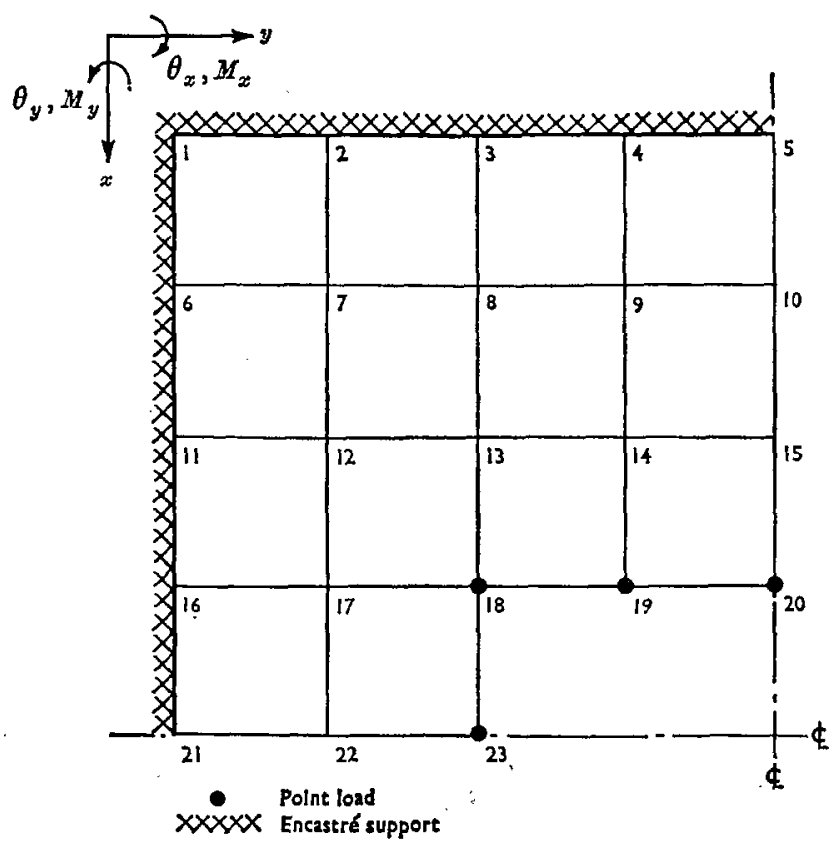

Fio. 8: TeST PROBLem

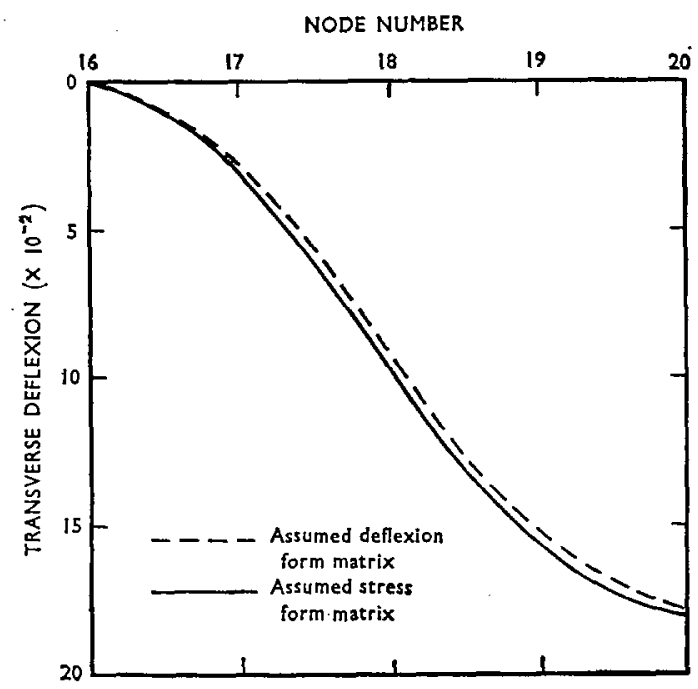

Fig. 9 


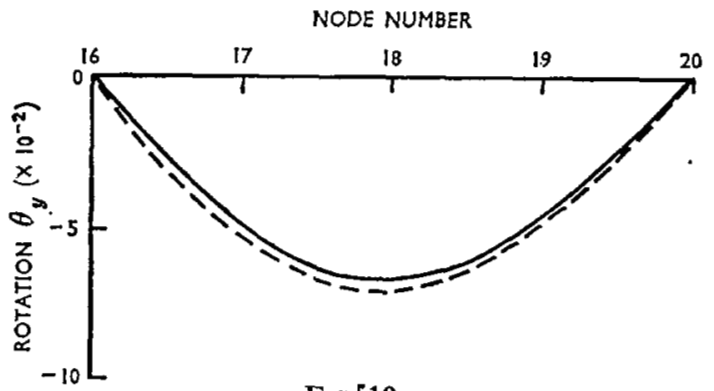

FIG:10

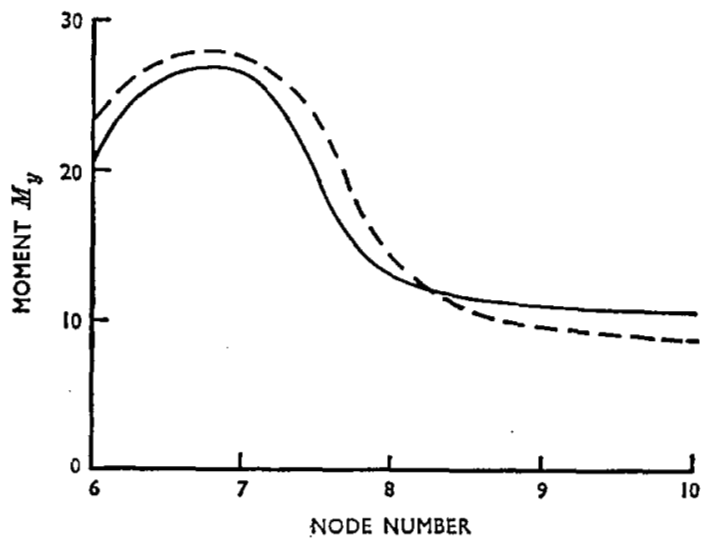

FIG. 11

distribution. It could then be queried why, in the Paper, the edge displacements were assumed to be in the form of a cubic polynomial which was similar to the displacement approach put forward by Melosh. ${ }^{\ominus}$ The two distinguishing factors were as follows.

(a) The Authors confined their assumed displacements to the edges of the element and said nothing of the overall deflected surface, whereas Melosh assumed that the functions varied linearly perpendicular to their dominant direction.

(b) As was properly emphasized in the Paper, equation (9c) ensured rotational compatibility across the boundaries of adjacent elements. Previous methods had not done this and the improvement in the consistency for values of moment at a mode, as a result of this, would be mentioned later in this contribution.

26. With regard to the direction from which convergency occurred it would appear preferable to approach the limit value from above. The major advantage of the finite element method was the ease with which complex and discontinuous structures could be analysed and thus, in such a case where no convenient cross-check can be made, the conservative design was necessary. 


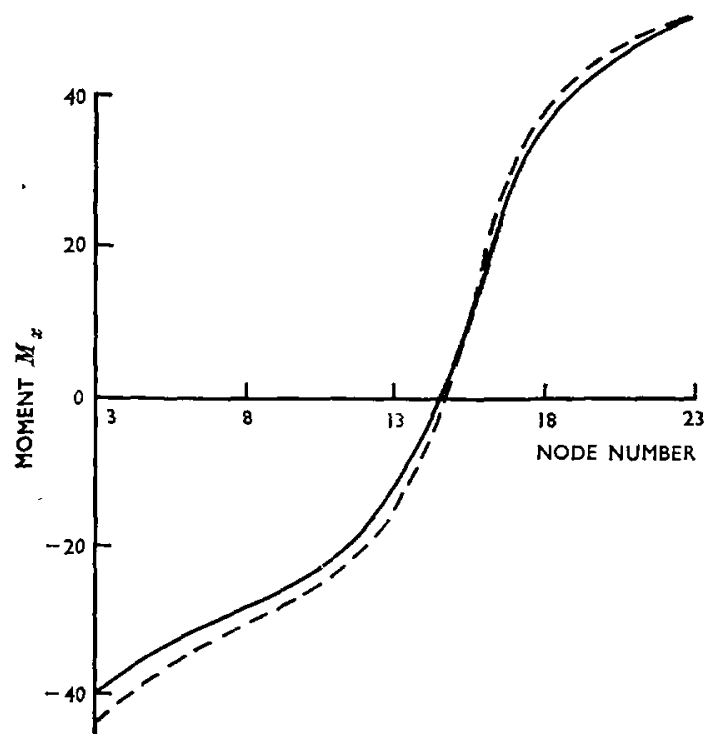

Fig. 12

27. The versatility of the triangular element in fitting non-rectangular outlines of a slab would seem to outweigh the poorish returns, notwithstanding that twice the number of triangular elements were employed than were needed for a rectangular mesh, and the property that convergency occurred from below the limit solution.

28. By using triangular elements a small saving in computer store space could usually be made and this could give the difference between being able to solve the problem as presented or having to reduce the naturally suggested mesh grading. The economical method of storing the assembled stiffness was as a column matrix composed of rows of half the band of the stiffness matrix.

29. The bandwidth of the stiffness matrix was given by $m=(p+1) 3$ for the triangular element, and $m=(p+2) 3$ for the rectangular element, where $p$ was the maximum number of nodes in any one row. Thus, the column matrix for a slab with a total of $n$ generalized nodal displacements occupied, $L=m(2 n-m+1) / 2$ locations.

30. Then for a rectangular slab with a $10 \times 10$ mesh grading the matrix $L$ occupied 13416 locations if rectangular elements were used but only 12438 locations when triangular elements were employed, representing a saving of $7 \%$ of working store.

31. As a further comparison of the two approaches in the finite element method a more complex slab had been analysed by the displacement method using the deflected form assumed by Zienkiewicz and Cheung ${ }^{2}$ and the stress distribution method expounded by the Authors (see Figs 8-12).

32. It should be noted that the values for generalized displacement calculated by the two methods were barely distinguishable. Those values for moments differed only slightly in view of the relatively coarse mesh and, in the case of $M_{y}$, the small absolute values of moment.

33. Finally, the Authors stated that the discontinuity in the values of moment at a node, depending on which element was considered in calculating the moment, was not greater than $1 \%$ of the maximum value of moment for the problems they considered. For the test problem (Fig. 8) the discontinuities were not unnaturally greatest for the moments with small absolute values. However, the variation in the 
values, about the mean, for moments in the medium range of those for the whole slab did not exceed $\pm 2.5 \%$ when derived by the assumed displacement method.

The Authors, in reply to Mr Smith, said that they agreed that their statement about convergence was too categorical. Subsequent experience had similarly shown that one could not be sure about the manner of convergence in the displacement approach. The Authors did not claim that their 'mixed' approach would always converge from below, but simply that it had always done so for problems where an exact solution was available for comparison.

35. Mr Smith's second point concerned discontinuities in bending moment between adjacent elements. These did occur in the 'mixed' method and in the displacement method of Zienkiewicz and Chang. Recently, it was true, new displacement functions had been proposed which removed the discontinuities. This was a big step forward.

36. Mr Tickell was correct in saying that the rule of matrix multiplication appeared to be violated throughout the Paper. What the Authors had done in presenting the Paper was to use curled brackets for both row and column vectors, letting the position in the equation determine whether a row or column vector was required in order to satisfy the rule of matrix multiplication.

\section{REFERENCES}

7. Hansteen H. Finite element displacement analysis of plate bending based on rectangular elements. Int. Symp. Use electron. digital Computers struct. Engng. Newcastle on Tyne, 1966.

8. SмIтн I. M. Some finite element and finite difference solutions to problems of bending of elastic plates and slabs. Res. Rep. Department of Civil Engineering, University of Glasgow, 1966.

9. Melosh R. J. A stiffness matrix for the analysis of thin plates in bending. J. Aero. Sci., 1961, 28, 34-42. 